Research Article

\title{
Immunohistochemical Findings in Idiopathic Inflammatory Bowel Disease in Nine Cats
}

\author{
María Cecilia Ricart, ${ }^{1}$ Silvia Feijóo, ${ }^{1}$ Giacomo Rossi $\mathbb{D}^{2},{ }^{2}$ Matteo Cerquetella, ${ }^{2}$ Víctor Castillo, \\ and Nélida Virginia Gómez \\ ${ }^{1}$ Universidad de Buenos Aires, Facultad de Ciencias Veterinarias, Cátedra de Clínica Médica de Pequeños Animales, \\ Av. Chorroarín 280, CP, C1427CWO Ciudad Autónoma de Buenos Aires, Argentina \\ ${ }^{2}$ School of Biosciences and Veterinary Medicine, University of Camerino, Via Circonvallazione 93/95-, 62024 Matelica (MC), Italy
}

Correspondence should be addressed to Giacomo Rossi; giacomo.rossi@unicam.it

Received 21 April 2020; Revised 3 September 2020; Accepted 22 September 2020; Published 1 October 2020

Academic Editor: Gerald J. Wyckoff

Copyright (c) 2020 María Cecilia Ricart et al. This is an open access article distributed under the Creative Commons Attribution License, which permits unrestricted use, distribution, and reproduction in any medium, provided the original work is properly cited.

\begin{abstract}
Inflammatory bowel disease is a term referring to a clinical condition characterized by recurrent/persistent gastrointestinal signs, which cannot be diagnosed only through histological findings, being an elimination diagnosis and needing therefore the previous exclusion of all other possible causes of gastrointestinal signs. The aim of the present study was to compare the expression of different cytokines on endoscopic biopsy samples of the small and large intestine of cats suffering from inflammatory bowel disease and healthy controls, by immunohistochemistry evaluation. Nine cats referred for chronic gastrointestinal signs to the Gastroenterology Service, Teaching Hospital, Faculty of Veterinary Sciences, University of Buenos Aires, were included. After being administered with antiparasitic drugs and after running complete laboratory exams, abdominal ultrasonography, etc., upper and lower gastrointestinal endoscopy with biopsy samplings for histopathology and immunohistochemistry was then performed. Controls were represented by archived samples from healthy cats (University of Camerino, Italy, Veterinary Pathology Unit Archive). On biopsy samples, the immunohistochemistry expression of the following antibodies was evaluated: TGF- $\beta, \mathrm{CD}^{+}$, FoxP3 ${ }^{+}$, TNF- $\alpha$, IL-1 $\beta$, IL-10, IL-12, and Th- $17^{+}$. Statistic analysis was performed with the nonparametric Mann-Whitney test and with Spearman test, considering significant $p<0.05$. TNF- $\alpha$, IL- $1 \beta$, IL-12, and $\mathrm{CD}^{+}$were significantly differently expressed between cats suffering from inflammatory bowel disease and controls, while no differences were found regarding TGF- $\beta$, IL-10, and FOXP3. A positive correlation was also found between proinflammatory cytokines and proinflammatory cytokines plus lymphocytes that were at the same time related to IL-10 and TGF- $\beta$. On the other hand, a negative correlation was found between proinflammatory and regulatory cytokines. Our results suggest an imbalance in the immune response which may play a role in the etiopathogenesis of feline inflammatory bowel disease.
\end{abstract}

\section{Introduction}

Inflammatory bowel disease (IBD) is a term referred in both dogs and cats to a clinical condition characterized by persistent or recurrent gastrointestinal (GI) signs (e.g., vomiting and diarrhea), chronic evolution (3-4 weeks), and histologic evidence of inflammation without any answer to empirical treatments, such as diet changes, or evidence of any other possible known cause [1-5]. Although there are no reliable data about its prevalence, due to the difficulty of achieving a definitive diagnosis, it is however considered a frequent condition in both dogs and cats [2]. It is the current opinion that this condition is favored by a genetic predisposition and the interaction between dietary and environmental factors and GI microbiota and that clinical signs result from an uncontrolled inflammatory response $[4,6]$. Indeed, although there are numerous theories on its pathogenesis, at the moment, a unique definition has not yet been reached [6].

For the abovementioned reasons, the diagnosis of IBD still remain a difficult achievement and the diagnostic path has been an object of interest worldwide in the last decades $[2,5]$. As an example, the messenger ribonucleic acid 
(mRNA) expression in intestinal biopsies of dogs presenting chronic enteropathy was studied, and measuring semiquantitatively the expression of determined cytokines, increased levels of interferon- (IFN-) $\gamma$, tumor necrosis factor- (TNF) $\alpha$, interleukin- (IL-) 2, IL-5, IL-12, and transforming growth factor- (TGF-) $\beta$ were found [7]. Such findings suggested the presence of a Th1 profile in dogs similar to the one found in man [8]. Similarly, other studies showed in dogs altered expression of cytokines in the small and large intestine with IBD $[8,9]$. However, other cytokine expression profiles through reverse transcriptase polymerase chain reaction (RT-PCR) were studied on biopsy samples which did not found differences between T-helper (Th)1 and Th2 [10-12]. In diseased patients (small bowel), an increased mRNA expression was found regarding Th1 (IL-2, IL-12, and INF$\gamma$ ) and Th2 (IL-5, TNF- $\alpha$ ) proinflammatory cytokines and the immunoregulatory cytokine TGF- $\beta$, which however does not represent a Th1- or Th2-specific profile, similarly to what was reported by the previously mentioned authors. In cases of IBD affecting the colon, the pattern can be considered quite similar being a higher presence of IL-2, IL-12, TNF- $\alpha$, and TGF- $\beta$ [13].

The aim of the present study was to compare, by immunohistochemistry (IHC) evaluation, the expression of different cytokines on endoscopic biopsy samples of the small and large intestine of cats diagnosed with inflammatory bowel disease and healthy controls, looking for possible markers for monitoring IBD.

\section{Materials and Methods}

2.1. Patients. The present one is a prospective study including nine cats referred for chronic gastrointestinal signs to the Gastroenterology Service, Teaching Hospital, Faculty of Veterinary Sciences, University of Buenos Aires, and diagnosed with IBD. All patients presented vomiting and/or diarrhea lasting for more than 3 weeks. All patients underwent fecal exams on three consecutive days and were treated with fenbendazole $50 \mathrm{mg} / \mathrm{kg}$ every $24 \mathrm{hrs}$, per os (PO), for three days, then for two more days after two weeks; hypoallergenic commerce diet was also administered. Complete blood count (CBC) and complete biochemistry, haemopathogens, thyroid function, and abdominal ultrasonography were also performed, plus any other exam deemed necessary based on the resulting data. Cats that did not respond to such empirical treatments and still presenting GI signs after 3 weeks were then subjected to upper and lower digestive endoscopy; biopsies of the gastric body, pyloric antrum, duodenum, and colon were taken.

Biopsies were preserved in $10 \%$ buffered formalin, and after $24 \mathrm{~h}$ of fixation, they were dehydrated through a complete alcohol series, then subsequently clarified in xylene and embedded in paraffin using an automatic processor. Paraffin blocks were cut in 3-4-micron-thick sections, then placed on slides and used for morphological evaluation (by hematoxylin and eosin stain) and immunohistochemistry. In all cats included in the study, an inflammatory infiltrate with morphological changes was found. All cats received immunosuppressants (i.e., prednisolone $1 \mathrm{mg} / \mathrm{kg}$ every 12 hours, PO, for one week, then tapered to $1 \mathrm{mg} / \mathrm{kg}$ every 24 hours, PO, for two-three weeks followed by $0.5 \mathrm{mg} / \mathrm{kg}$ every $24 \mathrm{hrs}$, PO, for two-three weeks, therefore continuing with the minimum effective dose) and hypoallergenic diet that was maintained for the whole study. All cats diminished clinical signs after treatment. Only the 9 cats complying with inclusion criteria and whose owners authorized inclusion were enrolled in the study.

Exclusion criterion was the necessity of performing a fullthickness biopsy of the GI tract, as occurred in patients with ultrasonographic findings of wall alteration involving all layers or submucosal layers and the presence of other causes justifying clinical signs of haemopathogens.

To obtain control tissue from healthy cats for this analysis, archived formalin-fixed and paraffin-embedded GI tissue samples from three cats (two males and one female) with no clinical signs of intestinal disease were retrieved from the University of Camerino, Italy, Veterinary Pathology Unit Archives. These samples had been obtained immediately postmortem from cats that were presented for euthanasia (euthanized cats (EC)) for severe injury due to car accidents. Ages ranged from 5 years to 13 years (mean 9.2 years), and histopathological examination of GI biopsies was normal in all cases.

2.2. Ethical Approval. The project was approved by the CICUAL (Comité Institucional de Cuidado y Uso de Animales de Experimentación) Faculty of Veterinary Sciences, University of Buenos Aires (project id. 2011/33; approved in the city of Buenos Aires, 12 October 2011). All owners signed informed consent.

2.3. Statistical Analysis. Statistical analysis was performed with GraphPad 5 program. For quantitative variable evaluation, the nonparametric Mann-Whitney test was used, while for the correlation between two quantitative variables, the Spearman test was used. Significant differences were those presenting $p<0.05$.

2.4. Immunohistochemistry. This technique was used to evaluate the expression of the following antibodies (Abs): TGF- $\beta$, $\mathrm{CD}^{+}{ }^{+}$, FoxP3 ${ }^{+}$, TNF- $\alpha$, IL-1 $\beta$, IL-10, IL-12, and Th- $17^{+}$. Sections embedded in paraffin were rehydrated and endogenous peroxidases neutralized with $3 \%$ hydrogen peroxide for 5 minutes, followed by a 5 -minute wash with distilled water.

For antigenic highlighting, the slides were preincubated with different retrieval solutions according to the suggestions indicated by the $\mathrm{Ab}$ producer: citrate buffer ( $\mathrm{pH}$ 6.0) for TGF- $\beta$, TNF- $\alpha$, and IL- $1 \beta$; EDTA ( $\mathrm{pH} 8.0$ ) for $\mathrm{CD}^{+}$and FoxP $3^{+}$; and $0.01 \mathrm{M}$ Tris-EDTA buffer (pH 9.0) for IL-12, IL-10, and Th-17, applied by immersion in the buffer solution and heating in a microwave oven (Black \& Decker, Towson, MD, USA) for 20 minutes or by incubation in a humid chamber. Nonspecific bindings were blocked by incubating the slides for 10 minutes with a protein-blocking agent (Dako, Carpinteria, CA, USA) before applying the primary $\mathrm{Ab}$. The slides were incubated overnight in a humid chamber with the primary Abs: monoclonal anti-human CD3 (moAb) (monoclonal rat anti-human clone MCA1477, Serotec AbD, Bio-Rad Laboratories, Hercules, CA, USA) diluted 1:50 in 
TBS buffer solution, moAb rat anti-mouse FoxP3 (clone FJK16s, eBioscience, San Diego, CA, USA) diluted 1:400, moAb mouse anti TGF- $\beta$ (clone 1D11, Serotec AbD, Bio-Rad Laboratories, Hercules, CA, USA) diluted $1: 25$, moAb mouse anti TNF- $\alpha$ (TNF706 + P/T2, Abcam, USA) diluted 1: 100, pAb rabbit-anti IL-1 $\beta$ (ab82558; Abcam, USA) diluted $1: 200$, pAb goat-anti feline IL-12 (AF1954- R \& D Systems, USA) diluted 1:50, pAb goat-anti feline IL-10 (AF736-SP$\mathrm{R} \& \mathrm{D}$ Systems, USA) diluted 1:50, and pAb rabbit anti Th-17 (Abcam, Cambridge, MA, 1:500-anti-ROR.). Antibodies were used to identify populations of $\mathrm{CD}^{+}$cells, $\mathrm{CD}$ Th- $17^{+}$cells, and other leukocyte subtypes producing the mentioned interleukins.

Immunoreaction with streptavidin-immunoperoxidase (streptavidin-immunoperoxidase, Black \& Decker, Towson, MD, USA) was visualized with 3,3-diaminobenzidine substrate (3,3'-diaminobenzidine substrate, Vector, Burlingame, UK). Cellular nucleus counterstain was performed with Mayer's hematoxylin. For negative immunohistochemical controls, primary antibodies were omitted. Cat spleen and tonsil sections were used for positive controls for TNF- $\alpha$, IL- $1 \beta$, IL-12, Th$17^{+}, \mathrm{CD}^{+}$and FoxP3 ${ }^{+}$cells. Cat placenta sections were used as control tissues for IL-10 and TGF- $\beta$ evaluation.

To perform the intestinal score of $\mathrm{CD}^{+} \mathrm{T}$ lymphocytes, FoxP $^{+}$, and $\mathrm{Th} 17^{+}$cells and to score all gastrointestinal cells producing TGF- $\beta$, TNF- $\alpha$, IL- $1 \beta$, IL-12, and IL-10, in different compartments of the duodenum and colon, consecutive cross sections of $3 \mu \mathrm{m}$ thickness were cut and placed consecutively on 8 separate slides, after which the ninth section was placed on the first slide, next to the first section, thus continuing for 40 sections. A single slide was analyzed for each cat with immunoreaction, which contained five cross sections. All cell types were evaluated using a 40x objective light microscope, a $10 \mathrm{x}$ eyepiece, and a square eyepiece grid $\left(10 \times 10\right.$ squares, with a total area of $\left.62,500 \mu \mathrm{m}^{2}\right)$. Ten appropriate fields were chosen for each compartment, and arithmetic means were calculated for each intestinal region. To avoid overvaluation in the count of all the determinations, those cells at the tissue margins were not considered.

The quantity of $\mathrm{CD}^{+}, \mathrm{FoxP}^{+}$, and $\mathrm{Th} 17^{+} \mathrm{T}$ cells and of cells producing TGF- $\beta$, TNF- $\alpha$, IL- $1 \beta$, IL-12, and IL-10 was calculated by using an image analysis system with an optical microscope (Carl Zeiss, Jena, Germany) equipped with a high-definition camera Javelin JE3462, a frame grabber Coreco Oculus OC-TCX, and a high-resolution monitor.

Color images were analyzed with Image-Pro Plus software (Media Cybernetics). Five transversal sections for each cat were recorded as well as the whole amount of immunoreactive T lymphocytes.

Then, for each cat, the total cellular amount, for cellular typology and for biopsy of the entire area, was calculated as the sum of all the examined areas of the specimen, in the consecutive 5 sections of a single slide. The results are expressed as immunohistochemistry- (IHC-) positive cells per $62,500 \mu \mathrm{m}^{2}$.

\section{Results and Discussion}

Considering clinical history, results of the complete work-up, histopathology, and response to anti-inflammatory treat- ment, nine cats were included in the study. Four were European common cats, three Siamese cats, and two Oriental cats; seven were males and two were females. At the time of biopsy samplings, one cat was 4 years old, three were 6 , one was 8 , one was 13, and three were 14 years old. Patients presented different clinical signs; the most frequent were vomiting and/or diarrhea, followed by hematochezia and presence of mucus; only one cat had weight loss. Four patients had a single clinical sign, four a combination of two signs, while the remaining one, three clinical signs. During the follow-up evaluation, signs of malnutrition, coat opacity, and worsening of the general condition were noticed.

No patients presented leukocytosis or alteration of the leukocyte formula; only one presented anemia (29\% hematocrit). Six cats did not present any alteration of the blood chemistry profile; the remaining three showed variable alterations of hepatic enzymes, however not indicative of the disease. Clinical biochemistry results are presented in Table 1.

All immunohistochemical evaluations, in healthy controls and IBD cats, were performed both in duodenal and colonic samples, and relative results are reported in Tables 2 and 3. A correlation between each determination in the duodenum was also performed, and results are showed in Table 4.

In the present study, European common cat represented the majority (4 out of 9 cats) of our patients. The possible higher presence of this disease in such group of animals had previously been noticed in patients of Teaching Hospital, Faculty of Veterinary Sciences, University of Buenos Aires [14]. However, in Argentina, purebred cats are not as frequent as in other countries, and the European common cat is the most prevalent. The remaining patients were Siamese cats and Oriental cats for which a breed predisposition to IBD is hypothesized [4]. The authors believe that the prevalence of male cats that was found in this study was only due to the randomness with which owners accepted to participate to the study completing diagnostic investigations; however, this should be considered a possible limitation of the study. According to literature, no sex predisposition for IBD is usually reported [3]. The most represented age was from 4 to 8 years (4 out of 9 cats), according to what was previously reported [4].

Clinically, always in accordance with previous reports [15], patients presented mainly vomiting and diarrhea, followed by hematochezia and lastly presence of mucus in feces and weight loss. Regarding the only cat that presented anemia, it could be due to chronic inflammation or chronic GI bleeding $[2,14]$, as no other cause was found during the work-up and follow-up. The absence of such alterations, although not indicative of the disease, could however be of help in excluding other diseases and moving on with the differential diagnosis. Similarly, also a negative biochemistry could be of help in eliminating other possible causes of clinical signs, routinely included in the differential list. Only few cats of the present study ( 3 out of 9) showed hematological signs of hepatic involvement that could be associated with IBD although not specific of its presence $[3,14]$.

The distribution of the different interleukins investigated furnished interesting data regarding their duodenal and 
TABLE 1: Clinical biochemistry results in the nine cats enrolled for the study: abnormal results are highlighted in bold.

\begin{tabular}{lcccccccc}
\hline & Alb & Total prot & Urea & Creatinine & ALT & AST & ALP & Phosphorus \\
\hline Cat 1 & 2.6 & $\mathbf{8 . 4}$ & 30 & 1.47 & $\mathbf{9 9}$ & 39 & 97 & 3.9 \\
Cat 2 & $\mathbf{2 . 2}$ & $\mathbf{6}$ & 53 & 1.7 & 73 & $\mathbf{8 3}$ & 50 & $\mathbf{2 . 6}$ \\
Cat 3 & 2.5 & $\mathbf{8 . 1}$ & 55 & 1.56 & $\mathbf{9 9}$ & $\mathbf{1 0 6}$ & 66 & 4.4 \\
Cat 4 & 2.5 & 7.5 & 60 & 1.84 & 52 & 23 & 93 & 3.8 \\
Cat 5 & 2.5 & 7.5 & 38 & 1.08 & 69 & 27 & 70 & 3.3 \\
Cat 6 & 2.5 & 7.4 & 64 & 1.71 & 50 & 39 & 3.7 \\
Cat 7 & 2.7 & 8 & 32 & 1.14 & 67 & 30 & 67 & 75 \\
Cat 8 & 2.8 & 7.7 & 45 & 1.25 & 70 & 62 & 3.5 \\
Cat 9 & 2.5 & 7.3 & 52 & 1.6 & 36 & 35 & 51 \\
\hline
\end{tabular}

Alb: albumin (mg/dl); Total prot: total protein (mg/dl); urea (mg/dl); creatinine (mg/dl); ALT: alanine aminotransferase (UI/l); AST: aspartate aminotransferase (mg/dl); ALP: alkaline phosphatase (mg/dl); phosphorus (mg/dl).

TABle 2: Duodenal immunohistochemistry-controls vs. diseased cats: distribution of each marker investigated in the duodenum of both healthy $(n=3)$ and cats suffering from IBD $(n=9)$. Results are expressed in median and range of cells positive to each IHC marker every $62,500 \mu \mathrm{m}^{2}$, considering 5 evaluation for each cat. Mann-Whitney test was then performed for statistical analysis.

\begin{tabular}{lcc}
\hline IHC & Controls $(n=15)$ & Diseased cats $(n=45)$ \\
\hline TGF- $\beta$ & $6(2-24)$ & $5(0-32)$ \\
TNF- $\alpha$ & $0(0-5)$ & $12(0-321)^{*}$ \\
IL-1 $\beta$ & $0(0-5)$ & $11(0-215)^{*}$ \\
IL-12 & $0(0-2)$ & $19(1-241)^{*}$ \\
IL-10 & $3(0-11)$ & $3(0-51)$ \\
Th-17 $^{+}$ & $0(0-0)$ & $8(1-121)^{\mathrm{N} / \mathrm{C}}$ \\
$\mathrm{CD}^{+}$ & $5(0-11)$ & $124.5(26-507)^{*}$ \\
FOXP3 $^{+}$ & $1(0-4)$ & $2(0-9)$ \\
\hline
\end{tabular}

${ }^{*} p<0.001 ;{ }^{\mathrm{N} / \mathrm{C}}$ not comparable.

TABle 3: Colonic immunohistochemistry-controls vs. diseased cats: distribution of each marker investigated in the colon of both healthy $(n=3)$ and cats suffering from IBD $(n=9)$. Results are expressed in median and range of cells positive to each IHC marker every $62,500 \mu \mathrm{m}^{2}$, considering 5 evaluation for each cat. Mann-Whitney test was then performed for statistical analysis.

\begin{tabular}{lcc}
\hline IHC & Control $(n=15)$ & Diseased cats $(n=45)$ \\
\hline TGF- $\beta$ & $4(1-19)$ & $3(0-38)$ \\
TNF- $\alpha$ & $1(0-7)$ & $15(0-371)^{*}$ \\
IL-1 $\beta$ & $1(0-7)$ & $9(0-246)^{*}$ \\
IL-12 & $1(0-4)$ & $14(2-324)^{*}$ \\
IL-10 & $2(0-13)$ & $7(0-33)$ \\
Th-17 $^{+}$ & $0(0-0)$ & $7(0-310)^{\mathrm{N} / \mathrm{C}}$ \\
$\mathrm{CD}^{+}$ & $2(0-9)$ & $161(32-495)^{*}$ \\
FOXP3 $^{+}$ & $1(0-2)$ & $1(0-9)$ \\
\hline
\end{tabular}

${ }^{*} p<0.001 ;{ }^{\mathrm{N} / \mathrm{C}}$ not comparable.

colonic location in cats with IBD. Interleukins and mediators' profiles have been more deeply investigated in dogs, and therefore, in literature, only little information is reported for cats. In studies performed in both dogs and cats, tech- niques usually used are fluorescence in situ hybridization (FISH) or PCR [10]. In this report, significant differences in the expression of TNF- $\alpha$, IL- $1 \beta$, and IL-12 both in the duodenum and in the colon are evidenced. These results agree with previous findings, performed by PCR, looking for mRNA expression of cytokines like IL-1, IL-12, and TNF- $\alpha$ which resulted in increased in cats with IBD $[16,17]$. All these interleukins are considered primarily proinflammatory, evidencing the process occurring in the gut during the disease. At the same time, also the presence of lymphocytes Th- $17^{+}$ was revealed in the duodenum and colon of diseased cats. This finding was not statistically comparable with healthy cats as these cells were not found in this group; such cells increased and were only found in cats with IBD, revealing inflammation. Finally, differences were also found for lymphocytes $\mathrm{CD}^{+}$that were increased in diseased patients, compared with healthy cats, showing that lymphocytes flow following inflammatory process. No statistical significance was found for TGF- $\beta$, IL-10, and FOXP3, suggesting an imbalance of the immune system in the etiology of the condition, as previously described [18] and being these interleukins mainly responsible for the inhibition of the abovementioned inflammatory process [2]. This inadequate inhibition would contribute to the following abnormal inflammatory response accompanied by the increase of inflammatory cytokines. Nevertheless, also an exaggerated immune response could justify these findings, as also reported in dogs and human medicine [19, 20].

Interestingly, other papers described increases of mRNA for IL-10 and TGF- $\beta[16,17]$, contrarily to what was showed by IHC with the present work. These differences could be not only due to the differences between the techniques used but also due to an altered mRNA transcript expression in cells or to a lack of cytokine release. It would be interesting to compare results of both techniques, performed on the same samples, to better define cytokines' role in inflamed tissues. In contrast to numerous studies that investigated mRNA expression changes in GI tissues of animals affected by IBD, a major strength of this work is the evaluation of cytokine changes in intestinal biopsies of affected cats. This is based on the idea that changes on protein transcripts can improve the understanding of underlying immune functionality, 
TABLE 4: Correlation among different IHC markers investigated in the duodenum: the table reports the " $r$ " of correlations among single IHC makers investigated in the duodenum of diseased cats. Spearman correlation was then performed for statistical analysis.

\begin{tabular}{lccccccc}
\hline IHC & TGF- $\beta$ & TNF- $\alpha$ & IL-1 $\beta$ & IL-12 & IL-10 & Th-17 & CD3 $^{+}$ \\
\hline TGF- $\beta$ & - & - & - & - & - & - & - \\
TNF- $\alpha$ & $-0.47^{*}$ & - & - & - & - & - & - \\
IL-1 $\beta$ & $-0.3^{*}$ & $0.78^{*}$ & - & - & - & - & - \\
IL-12 & $-0.32^{*}$ & $0.79^{*}$ & $0.84^{*}$ & - & - & - & - \\
IL-10 & $0.52^{*}$ & $-0.33^{*}$ & -0.11 & -0.07 & - & - & - \\
Th-17 $^{+}$ & $-0.6^{*}$ & $0.75^{*}$ & $0.67^{*}$ & $0.73^{*}$ & $-0.4^{*}$ & - & - \\
CD3 $^{+}$ & $-0.26^{*}$ & $0.67^{*}$ & $0.71^{*}$ & $0.79^{*}$ & 0.79 & $0.69^{*}$ & - \\
FOXP3 $^{+}$ & $0.3^{*}$ & $0.23^{* *}$ & $0.24^{* *}$ & $0.21^{* *}$ & $0.21^{* *}$ & -0.13 & -0.13 \\
\hline
\end{tabular}

${ }^{*} p<0.001,{ }^{* *} p<0.01$.

better than genome levels. To our knowledge, there are only some experimental studies that evaluated protein expression and mRNA expression levels in intestinal biopsies of IBDaffected cats [17]. In dogs, Kołodziejska-Sawerska et al. demonstrated similar upregulation of TNF- $\alpha$, TGF- $\beta$, IL$1 \beta$, IL-4, and IL-10 mRNA levels in intestinal biopsies and in blood [21].

The missing mRNA analysis might thereby be seen as a limitation of our work, but it is known that mRNA levels do not necessarily correlate with protein levels. Particularly, IL-10 is known to underlie posttranscriptional regulation processes articulately influencing later protein levels [22]. This might explain the diverse data concerning IL-10 expression levels in this series. One of the main advantages in studying gene expression profiles is the detection of very early transcriptome changes, but, in clinical studies, the levels of expression of different cytokine transcripts are directly related to the outcome of the disease and clinical signs.

The present manuscript showed a highly significant positive correlation among proinflammatory cytokines, accordingly to what was previously described by other authors. A moderate positive correlation was also evidenced between proinflammatory cytokines and lymphocytes and between IL-10 and TGF- $\beta$. Contrariwise, a weak negative correlation between proinflammatory and regulatory cytokines was found. Our results support the hypothesis that in the etiology of IBD, an imbalance of the immune response could be involved, as result of a growth of proinflammatory mediators and inflammatory cells and a lack of increase in regulatory cells.

\section{Conclusions}

In the present study, both proinflammatory cytokines (TNF$\alpha, \mathrm{IL}-1 \beta$, and IL-12) and $\mathrm{CD} 3^{+}$cells resulted to be increased in cats with IBD, in both the duodenum and colon, when compared to controls. No significant changes were found for regulatory cytokines investigated. Further studies are needed to define whether such modifications could be present also in GI diseases other than IBD or if they are characteristic of this condition and if it could be sufficient to perform these IHC evaluations in a single GI endoscopy (upper or lower), and not in two, to differentiate diseased cats from healthy ones.

\section{Data Availability}

The data used to support the findings of this study are included within the article.

\section{Conflicts of Interest}

The authors declare no conflict of interest regarding the present manuscript.

\section{Acknowledgments}

The present study was realized with the subsidy of the University of Buenos Aires, code UBACyT: 20020100100002.

\section{References}

[1] M. Cerquetella, A. Spaterna, F. Laus et al., "Inflammatory bowel disease in the dog: differences and similarities with humans," World Journal of Gastroenterology, vol. 16, no. 9, pp. 1050-1056, 2010.

[2] R. J. Washabau, M. J. Day, M. D. Willard et al., "Endoscopic, biopsy, and histopathologic guidelines for the evaluation of gastrointestinal inflammation in companion animals," Journal of Veterinary Internal Medicine, vol. 24, no. 1, pp. 10-26, 2010.

[3] A. E. Jergens, J. M. Crandell, R. Evans, M. Ackermann, K. G. Miles, and C. Wang, "A clinical index for disease activity in cats with chronic enteropathy," Journal of Veterinary Internal Medicine, vol. 24, no. 5, pp. 1027-1033, 2010.

[4] A. E. Jergens, "Feline idiopathic inflammatory bowel disease," Journal of Feline Medicine and Surgery, vol. 14, no. 7, pp. 445458, 2012.

[5] M. Cerquetella, G. Rossi, J. S. Suchodolski et al., "Proposal for rational antibacterial use in the diagnosis and treatment of dogs with chronic diarrhoea," Journal of Small Animal Practice, vol. 61, no. 4, pp. 211-215, 2020.

[6] J. R. S. Dandrieux, "Inflammatory bowel disease versus chronic enteropathy in dogs: are they one and the same?," Journal of Small Animal Practice, vol. 57, no. 11, pp. 589-599, 2016.

[7] K. Allenspach, "Clinical immunology and immunopathology of the canine and feline intestine," Veterinary Clinics of North 
America: Small Animal Practice, vol. 41, no. 2, pp. 345-360, 2011.

[8] A. J. German, C. R. Helps, E. J. Hall, and M. J. Day, "Cytokine mRNA expression in mucosal biopsies from German shepherd dogs with small intestinal enteropathies," Digestive Diseases Sciences, vol. 45, no. 1, pp. 7-17, 2000.

[9] T. M. McCann, A. E. Ridyard, R. W. Else, and J. W. Simpson, "Evaluation of disease activity markers in dogs with idiopathic inflammatory bowel disease," Journal of Small Animal Practice, vol. 48, no. 11, pp. 620-625, 2007.

[10] I. R. Peters, C. R. Helps, E. L. Calvert, E. J. Hall, and M. J. Day, "Cytokine mRNA quantification in duodenal mucosa from dogs with chronic enteropathies by real-time reverse transcriptase polymerase chain reaction," Journal of Veterinary Internal Medicine, vol. 19, no. 5, pp. 644-653, 2005.

[11] S. N. Sauter, K. Allenspach, F. Gaschen, A. Gröne, E. Intsouka, and J. W. Blum, "Cytokine expression in an ex vivo culture system of duodenal samples from dogs with chronic enteropathies: modulation by probiotic bacteria," Domestic Animal Endocrinology, vol. 29, no. 4, pp. 605-622, 2005.

[12] N. M. Schreiner, F. Gaschen, A. Gröne, S. N. Sauter, and K. Allenspach, "Clinical signs, histology, and CD3-positive cells before and after treatment of dogs with chronic enteropathies," Journal of Veterinary Internal Medicine, vol. 22, no. 5, pp. 1079-1083, 2008.

[13] A. E. Ridyard, T. J. Nuttall, R. W. Else, J. W. Simpson, and H. R. P. Miller, "Evaluation of Th1, Th2 and immunosuppressive cytokine mRNA expression within the colonic mucosa of dogs with idiopathic lymphocytic-plasmacytic colitis," Veterinary Immunology and Immunopathology, vol. 86, no. 3-4, pp. 205-214, 2002.

[14] S. Feijoó, "Inflammatory bowel disease. Diagnostic path and treatment. Differential diagnosis and therapeutical management of vomiting patients," in Proceeding of LAVC, Latin American Veterinary Conference, pp. 56-67, Lima, Perú, October 2008.

[15] S. R. Hauck, K. Gisselman, A. Cordner, and A. G. Nicholson, "Chronic vomiting in cats: etiology and diagnostic testing," Journal American Animal Hospital Association, vol. 52, no. 5, pp. 269-276, 2016.

[16] N. Nguyen Van, K. Taglinger, C. R. Helps, S. Tasker, T. J. Gruffydd-Jones, and M. J. Day, "Measurement of cytokine mRNA expression in intestinal biopsies of cats with inflammatory enteropathy using quantitative real-time RT-PCR," Veterinary Immunology and Immunopathology, vol. 113, pp. 404-414, 2006.

[17] S. Janeczko, D. Atwater, E. Bogel et al., "The relationship of mucosal bacteria to duodenal histopathology, cytokine mRNA, and clinical disease activity in cats with inflammatory bowel disease," Veterinary Microbiology, vol. 128, no. 1-2, pp. 178-193, 2008.

[18] C. Pedros, F. Duguet, A. Saoudi, and M. Chabod, "Disrupted regulatory T cell homeostasis in inflammatory bowel diseases," World Journal of Gastroenterology, vol. 22, no. 3, pp. 974-995, 2016.

[19] M. Chichlowski and L. P. Hale, "Bacterial-mucosal interactions in inflammatory bowel disease-an alliance gone bad," American Journal of Physiology-Gastrointestinal and Liver Physiology, vol. 295, no. 6, pp. G1139-G1149, 2008.

[20] R. B. Sartor, "Mechanisms of disease: pathogenesis of Crohn's disease and ulcerative colitis," Nature Clinical Practice Gastroenterology and Hepatology, vol. 3, no. 7, pp. 390-407, 2006.
[21] A. Kołodziejska-Sawerska, A. Rychlik, A. Depta, M. Wdowiak, M. Nowicki, and M. Kander, "Cytokines in canine inflammatory bowel disease," Polish Journal of Veterinary Sciences, vol. 16, no. 1, pp. 165-171, 2013.

[22] W. Sipos, C. Duvigneau, F. Sterz et al., "Changes in interleukin-10 mRNA expression are predictive for 9-day survival of pigs in an emergency preservation and resuscitation model," Resuscitation, vol. 81, no. 5, pp. 603-608, 2010. 